\title{
Mobility management schemes and mobility issues in low power wireless sensor network
}

\begin{abstract}
One of the current most significant active research areas in wireless communication technologies is Wireless Sensor Network (WSN). WSN is widely adopted in the ubiquitous networking due to its large applications in different fields such as healthcare, agriculture, livestock monitoring, and etc. WSN is labeled as less reliable due to few drawbacks such as energy consumption, networking cost, mobility management, and etc. The mobility management issue was given less attention in the WSN because the significant of seamless connectivity is underestimated. Based on analysis of recent literatures, our paper seeks to address the mobility issues in WSN. This paper gives an overview of the available mobility management schemes and examines mobility issues of nodes in network-based mobility schemes.
\end{abstract}

Keyword: Wireless sensor network; Mobility; Handoff; Mobility issues 